\title{
An automatic collector to monitor insoluble atmospheric deposition: an application for mineral dust deposition
}

B. Laurent et al.

Correspondence to: B. Laurent (benoit.laurent@lisa.u-pec.fr) 
1 Technical notes about the CARAGA collector.

2

3 In order to transport and implement easily the CARAGA collector on remote site, it has been

4 designed as separate modules, electrically connected to one another by tight connections.

6 The 7 modules of the $C A R A G A$ are:

7

8 - A tripod which can be removed and adjusted (height and horizontal level) to insure the 9 correct installation of the sampling unit.

10 - A spacer set to make higher the top of the collecting funnel ( $2.5 \mathrm{~m}$ above the ground) and 11 allowing fixing the others modules.

12 - An electrical control unit containing the battery and the regulator system and allowing to 13 program operation commands. The device allows testing the collector functions and 14 simulating a complete cycle of the collector running. The program can be adjusted on-site 15 (selection of day and time for the sampling time step, duration of the workflow...).

16 - An automated sampling rotating unit (carousel) of 25-filter holders in which the drive unit, 17 the enslavement and the filtered air ventilation system are installed.

18 - The collecting top part which consists in a graphite funnel $\left(0.2 \mathrm{~m}^{2}\right)$ equipped with vibrating 19 and rinsing systems and a casing protection.

20 - A reservoir containing ultrapure water to rinse automatically the funnel and the sample 21 system.

22 - A ladder hinged on the tripod and the spacer set allowing accessing and maintaining the 23 modules.

24 - A solar panel $20 \mathrm{~W}$.

26 The power supply is possible in $12 \mathrm{Vdc}$ or $24 \mathrm{Vdc}$ and the average consumption is 40 to 45 $27 \mathrm{~mA} \mathrm{~h}^{-1}$. The CARAGA has an overall mass of $100 \mathrm{~kg}$. It is necessary to provide stowage and 28 slings to fix the CARAGA on the ground. 